\title{
Lumbar plexopathy as a complication of percutaneous nephrolithotomy in a horseshoe kidney
}

\author{
Andrea G. Lantz, MD, FRCSC, $R$. John D'A Honey, MD, FRCSC ${ }^{\dagger}$ \\ *Department of Urology, Dalhousie University, Halifax, NS; † Division of Urology, Department of Surgery, University of Toronto, Toronto, ON
}

Cite as: Can Urol Assoc J 2015;9(1-2):e78-80. http://dx.doi.org/10.5489/cuaj.2468

Published online February 9, 2015.

\section{Abstract}

Treatment of nephrolithiasis in horseshoe kidneys can be challenging due to anomalies in renal position, collecting system anatomy and vascular supply. We report on a patient who was referred after a failed percutaneous nephrolithotomy for a left moiety staghorn calculus in a horseshoe kidney. Two punctures had been performed involving upper and middle posterior calyces. Both were very medially placed and inadvertently traversed the psoas muscle, resulting in lumbar plexopathy with permanent deficit. This complication presented postoperatively with left leg weakness, paresthesia, and pain which impaired independent ambulation. The patient went on to be successfully treated for her stone disease with robotic-assisted laparoscopic pyelolithotomy.

\section{Introduction}

Horseshoe kidney is the most prevalent renal fusion anomaly and occurs in $0.25 \%$ of the general population. Horseshoe kidneys have anomalies related to position, orientation of the collecting system, and anomalies of the renal pelvis and ureteropelvic junction, which alters the surgical approach of urolithiasis. ${ }^{1}$ Percutaneous nephrolithotomy (PCNL) is effective in managing complex urolithiasis in horseshoe kidneys. ${ }^{2-4}$ The access is often more inferior and medial than usual due to the decreased renal ascent and rotation anomalies. ${ }^{5}$ Complications of PCNL may include bleeding, urinary tract infection, urinary fistula, bowel injuries, and injury to adjacent organs, such as the spleen, liver, and pleura. ${ }^{6}$ Due to the malrotation, many of the calyces point directly posteriorly or postero-laterally, making access surprisingly simple. As the isthmus is situated anterior to the aorta, the antero-posterior angle of axis is exaggerated, allowing a very direct view down the axis of the kidney from the upper pole. However, this exaggerated renal axis means that an access through a mid-calyx can result in a long tract with very little maneuverability due to the fixed lower pole.

We report a previously unreported complication following PCNL for a staghorn calculus in a horseshoe kidney.

\section{Case report}

A 63-year-old woman with a history of urolithiasis and spontaneous stone passage was referred following a failed left PCNL in 2009, complicated by a lumbar plexopathy. She had bilateral staghorn calculi in a horseshoe kidney after workup for intermittent flank pain. Computed tomography (CT) showed a horseshoe kidney with the right moiety containing a $3.4 \times 3.9 \times 2-\mathrm{cm}$ staghorn calculus with thinning of the renal parenchyma and lower pole hydronephrosis. The left moiety contained a $1.5 \times 1.4 \times 2.5-\mathrm{cm}$ staghorn calculus with preserved parenchyma and no hydronephrosis. The patient's comorbidities included non-insulin dependent diabetes, sarcoidosis, and hypercholesterolemia.

During the complicated left PCNL, an upper pole puncture was initially used; however, due to the long infundibulum, the renal pelvis and most of the stone could not be accessed. A second tract was inserted in a middle calyx and again the bulk of stone could not be treated due to poor visibility and inability to reach the stone. A nephroureterostomy tube was placed through the middle calyx. A $5 F r$ open-ended ureteral catheter was left through the upper pole tract, which was removed on postoperative day 1 . The procedure was abandoned.

On postoperative day 1 , the patient complained of left leg weakness and paresthesia along the medial aspect of the upper and lower leg. Neurosurgical consultation was sought and a CT scan was performed. The CT scan showed that the nephroureterostomy tube entered the back just left of the midline at the level of $L 3$, passed over the left transverse process of $L 4$, and through the medial aspect of the left psoas muscle traveling in close proximity to the exiting left L3 nerve root (Fig. 1). The nephroureterostomy tube was removed. A ureteral stent was subsequently placed due to 

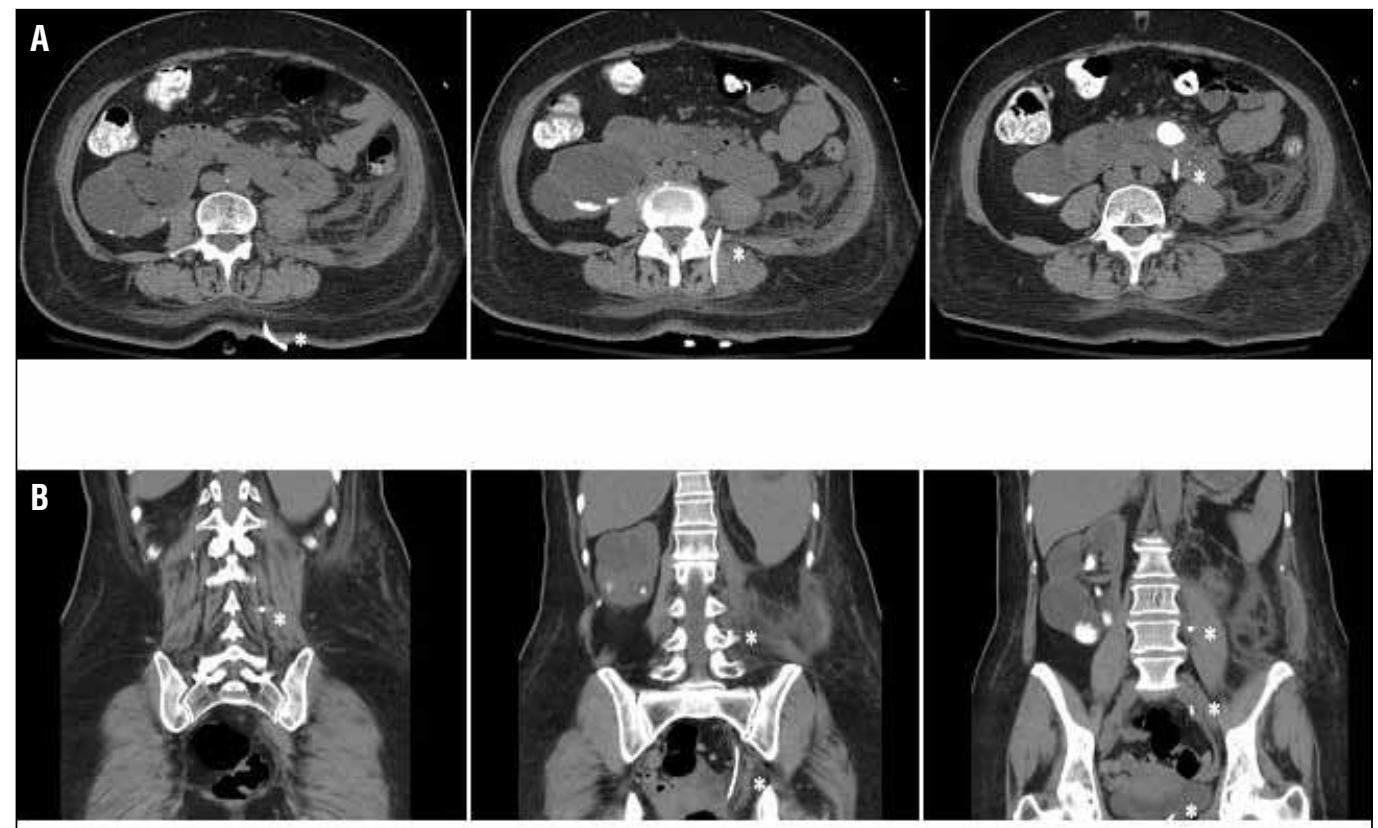

Fig. 1. A: Axial views; ${ }^{*}$ nephroureteral tube. B: Coronal views; ${ }^{*}$ nephroureteral tube.

obstruction and increasing serum creatinine. The patient received aggressive physiotherapy treatment and was discharged on postoperative day 10 , ambulating using a walker. She was referred for a second opinion.

Electromyographic (EMG) studies revealed a left lumbar plexopathy and denervation in the left L1, L2, L3, and L4 myotomes, predominantly affecting the L2 and L3 myotomes. Clinically, the patient complained of left leg pain and showed atrophy of the vastus lateralis muscle, weakness of hip flexion, hip adduction, knee extension, absent patellar reflex, and decreased light touch and pinprick sensation to the medial thigh and lower leg. One year after the injury, follow-up EMG studies showed improvement in electrophys-

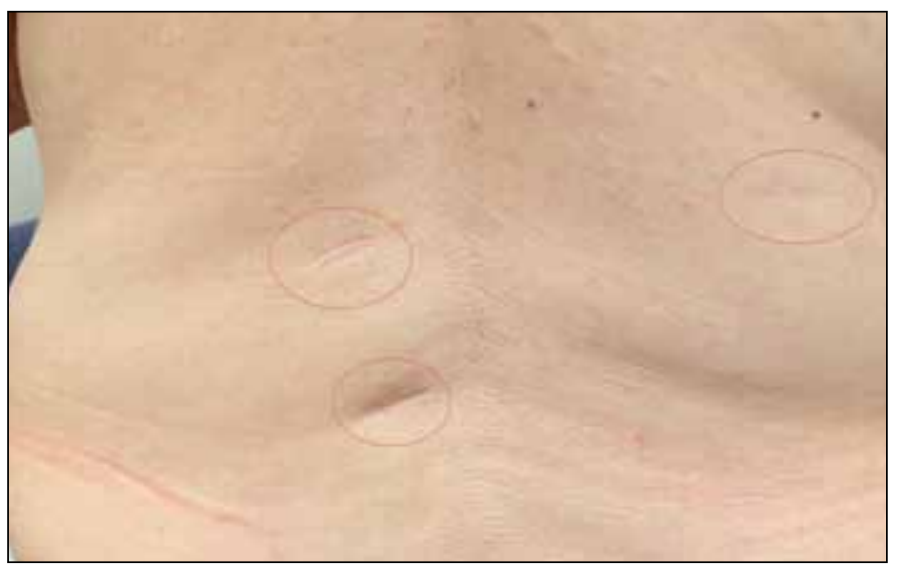

Fig. 2. Left access tracts traversing paraspinal muscles, right access tract lateral to paraspinal muscles. iologic parameters and evidence of re-innervation. Her pain was decreased and some gain in strength was noted. She was ambulating with a cane, but unable to return to work.

After referral to the senior author, robotic-assisted laparoscopic pyelolithtomy was recommended for the left moiety. The patient was reluctant to have any further invasive treatment, but after failed shockwave lithotripsy she agreed to a robotic approach. Subsequently, a robotic-assisted laparoscopic pyelolithotomy was performed resulting in stone-free status in that left moiety. An uncomplicated right PCNL was later performed (Fig. 2).

\section{Discussion}

PCNL to treat urolithiasis is a useful and effective approach in anomoalous kidneys ${ }^{2-4,7-9}$ Horseshoe kidneys are prone to urolithiasis due to stasis from ureteropelvic junction abnormalities ${ }^{1}$ infection, and metabolic derangements. ${ }^{10}$ Challenges with performing PCNL in horseshoe kidneys relates to the abnormalities in renal position and malrotation. Access through the lower pole should be avoided because of the very long, medially placed tract with little maneuverability and potential interference by the posterior iliac crest during rigid nephroscopy. Horseshoe kidneys are less mobile than other kidneys ${ }^{1}$ and may be prone to parenchymal tears and bleeding if excessive torque is placed on the nephroscope.

Neuropathy is a rare complication following PCNL; it has not been reported after PCNL for horseshoe kidneys. To our 
knowledge, the only previously reported lumbar neuropathy due to PCNL was reported by Monga and colleagues. ${ }^{11}$ In that report, a 29-year-old woman suffered a femoral neuropathy during a left PCNL to remove an encrusted stent in her pelvic kidney. Neuropathy in this case was attributed to direct trauma to the dorsal divisions of the lumbar plexus along the psoas muscle.

\section{Conclusion}

PCNL is effective to treat urolithiasis in horseshoe kidneys; however, care must be taken during the surgical planning due to the anomalous anatomy inherent to horseshoe kidneys. An upper pole access should be chosen whenever possible. One must be cognizant of the adjacent anatomy and ensure calyceal puncture and tract dilatation are not too far medial, which poses a risk for lumbar plexopathy as discussed in this case. If a safe access tract is not feasible, alternative methods, such as laparoscopic or robotic-assisted pyelolithotomy, should be considered.

Competing interests: Dr. Lantz declares no competing financial or personal interests. Dr. Honey has received grants from Cook Medical in the form of a partially funded Endourology fellowship.

This paper has been peer-reviewed.

\section{References}

1. Cussenot 0 , Desgrandchamps $F, 0$ llier $P$, et al. Anatomical bases of percutaneous surgery for calculi in horseshoe kidney. Surg Radiol Anat 1992;14:209-13. http://dx.doi.org/10.1007/BF01794940

2. Ozden E, Bilen CY, Mercimek MN, et al. Horseshoe kidney: Does it really have any negative impact on surgical outcomes of percutaneous nephrolithotomy? Urology 2010;75:1049-52. http://dx.doi. org/10.1016/i.urology.2009.08.054

3. Raj GV, Auge BK, Weizer AZ, et al. Percutaneous management of calculi within horseshoe kidneys. J Urol 2003;170:48-51. http://dx.doi.org/10.1097/01.ju.0000067620.60404.2d

4. Liatsikos EN, Kallidonis P, Stolzenburg JU, et al. Percutaneous management of staghorn calculi in horseshoe kidneys: A multi-institutional experience. J Endourol 2010;24:531-6. http://dx.doi.org/10.1089/ end.2009.0264

5. Miller NL, Matlaga BR, Handa SE, et al. The presence of horseshoe kidney does not affect the outcome of percutaneous nephrolithotomy. J Endourol 2008;22:1219-26. http://dx.doi.org/10.1089/ end.2008.0051

6. Michel MS, Trojan L, Rassweiler JJ. Complications in percutaneous nephrolithotomy. Eur Urol 2007;51:899906. http://dx.doi.org/10.1016/i.eururo.2006.10.020

7. Osther PJ, Razvi H, Liatsikos E, et al. Percutaneous nephrolithotomy among patients with renal anomalies: Patient characteristics and outcomes; A subgroup analysis of the clinical research office of the Endourological Society Global Percutaneous Nephrolithotomy Study. J Endourol 2011;25:1627-32. http://dx.doi. org/10.1089/end.2011.0146

8. Rana AM, Bhojwani JP. Percutaneous nephrolithotomy in renal anomalies of fusion, ectopia, rotation, hypoplasia, and pelvicalyceal aberration: Uniformity in heterogeneity. J Endourol 2009;23:609-14. http://dx.doi.org/10.1089/end.2007.0430

9. Gupta NP, Mishra S, Seth A, et al. Percutaneous nephrolithotomy in abnormal kidneys: Single-center experience. Urology 2009;73:710-4. http://dx.doi.org/10.1016/j.urology.2008.10.070

10. Raj GV, Auge BK, Assimos D, et al. Metabolic abnormalities associated with renal calculi in patients with horseshoe kidneys. J Endourol 2004;18:157-61. http://dx.doi.org/10.1089/089277904322959798

11. Monga M, Castaneda-Zuniga WR, Thomas R. Femoral neuropathy following percutaneous nephrolithotomy of a pelvic kidney. Urology 1995;45:1059-61. http://dx.doi.org/10.1016/S0090-4295(99)80133-2

Correspondence: Dr. Andrea G. Lantz, Department of Urology, Dalhousie University, Halifax, NS; andrea.lantz@gmail.com 